\title{
Lithographic Tuning of a Two-Dimensional Photonic Crystal Laser Array
}

\author{
O. Painter, A. Husain, A. Scherer, P. T. Lee, I. Kim, J. D. O’Brien, and P. D. Dapkus, Fellow, IEEE
}

\begin{abstract}
One attraction of photonic crystals is the ability to control optical device characterstics by lithographically varying the geometry. In this letter, we demonstrate a $10 \times 10$ array of optically pumped two-dimensional (2-D) photonic crystal defect lasers with varying lattice parameters. By adjusting the photonic crystal interhole spacing as well as the hole diameter we are able to tune the laser wavelength from 1500 to $1625 \mathrm{~nm}$ on a monolithic InP-InGaAsP wafer. A wavelength resolution of $10 \mathrm{~nm}$ from device to device was obtainable, limited by the lithography and etching tolerances of our fabrication method.
\end{abstract}

Index Terms-InGaAsP, laser array, microcavities, photonic crystals, quantum-well laser, semiconductor device fabrication.

\section{INTRODUCTION}

$\mathbf{T}$ HE use of anistropic etching techniques to form high-contrast periodic dielectric structures in which light is strongly Bragg reflected over a large angular range has been an active field of research since the first proposal made by Yablonovitch in 1987 [1] of controllable spontaneous emission. Photonic crystals produce strong disperion of electromagnetic waves and can be engineered to diffract, guide, or trap light [2]-[4] in spatial dimensions on the order of the wavelength of light itself. In this letter, we present recent work on lasers formed from two-dimensional (2-D) photonic crystal single defect cavities embedded in a half-wavelength thick waveguide. Simple lithographic adjustments in the cavity geometry can be used to control the wavelength, emission direction, and polarization of cavity modes [5]. The combination of high device density, and lithographically controllable light emission make this technology interesting for a variety of laser/detector array applications such as beam-steering, beam-shaping, and multiwavelength optical signal processing.

A schematic of the single defect laser cavity is shown in Fig. 1. The 2-D photonic crystal consists of a triangular array of etched air holes in a half-wavelength dielectric slab of refractive index 3.4. For an index contrast of 3.4:1 and for suitably large hole radius to lattice spacing ratio $(r / a)$ a photonic bandgap for guided modes opens up [5]. The removal of a single hole forms a local energy well for photons, and energy is trapped in the cavity by distributed Bragg reflection off of the 2-D photonic crystal, and total internal reflection within the high refractive index slab

Manuscript received March 27, 2000; revised July 1, 2000.

O. Painter, A. Husain, and A. Scherer are with the Department of Electrical Engineering, California Institute of Technology, Pasadena, CA 91125 USA (e-mail: opainter@its.caltech.edu).

P. T. Lee, I. Kim, J. D. O'Brien, and P. D. Dapkus are with the Department of Electrical Engineering-Electrophysics, University of Southern California, Los Angeles, CA 90089-0271 USA.

Publisher Item Identifier S 1041-1135(00)07873-3.

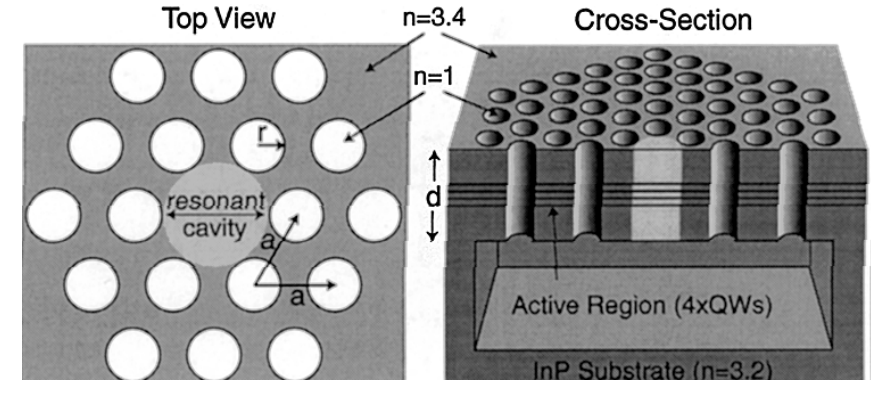

Fig. 1. Illustration of the photonic crystal microcavity. The optical cavity consists of a 2-D triangular array of air holes in a half-wavelength-thick suspended membrane. A single hole is removed in order to form a resonant cavity in the photonic crystal.

waveguide. A number of high- $Q$ cavity modes form within the frequency bandgap of the photonic crystal host [6]. These cavity modes are highly sensitive to the local photonic crystal environment and can be wavelength tuned by adjusting the host crystal dimensions.

\section{WaVElength TUNing PARAMETERS}

In the photonic crystal studied here we have three geometric tuning parameters: lattice spacing $a$, hole radius $r$, and slab thickness $d$. In order to understand the tuning affects of the photonic crystal geometry on the cavity modes it is most convenient to normalize all structural parameters to the interhole spacing, $a$. The photonic crystal slab is then described by its normalized hole radius, $r / a$, and its normalized slab thickness, $d / a$. Due to the scaling properties of Maxwell's equations a normalized eigenfrequency can also be defined

$$
\omega^{n}=\frac{a \omega}{2 \pi c}=\frac{a}{\lambda_{o}}
$$

where the $\omega$ is the angular frequency, $c$ is the speed of light, $\lambda_{o}$ is the wavelength in vacuum, and the superscript $n$ denotes normalized frequency. For fixed $r / a$ and $d / a$ the wavelength simply scales with lattice spacing.

Since the waveguide thickness of the laser cavities is fixed in our experiment, the ratio $d / a$ will vary with lattice spacing. The effects of the vertical waveguiding on the photonic crystal slab modes can be approximated by separating the inplane and vertical components of momentum

$$
\omega^{2} \approx \omega_{2 \mathrm{D}}^{2}+\left(\frac{c}{n_{\mathrm{eff}}^{z}}\right)^{2} k_{z}^{2}
$$

where $\omega_{2 \mathrm{D}}$ is the mode frequency in the 2-D case (inplane curvature only), $k_{z} \equiv 2 \pi / \lambda_{z}$ represents the curvature due to the 
vertical guiding, and $n_{\mathrm{eff}}^{z}$ is an effective index determined by the field pattern of the 2-D mode and its overlap with dielectric material. For the fundamental vertically guided modes of the photonic crystal slab, assuming strong confinement, $\lambda_{z} \approx 2 d$. The frequency of a mode in the photonic crystal waveguide, after normalization using (1), can then be written as

$$
\omega^{n} \approx \sqrt{\left(\omega_{2 \mathrm{D}}^{n}\right)^{2}+\left(\frac{1}{2 n_{\mathrm{eff}}^{z}}\right)^{2}\left(\frac{d}{a}\right)^{-2}}
$$

Thus for fixed $r / a$ and fixed $d$ the normalized frequency increases with increasing lattice spacing and the wavelength varies sublinearly with lattice spacing.

The variation of the mode frequency with $r / a$ is more complicated, however on an intuitive level one may argue that as $r / a$ increases the mode frequency will also increase due to the reduced filling fraction of high dielectric material [7]. In the next section we vary all three parameters $a, d / a$, and $r / a$ in the laser cavities in order to tune the wavelength of the modes of the single defect photonic crystal cavities.

\section{Measurement Results AND Discussion}

The epitaxy for the photonic crystal laser cavities was grown by metal-organic chemical vapor deposition (MOCVD) on InP, and contains four $0.85 \%$ compressively strained quaternary quantum wells [8] designed for a peak emission wavelength of $1.55 \mu \mathrm{m}$ at room temperature. The final waveguide thickness is $211 \mathrm{~nm}$, which is approximately a half-wavelength at 1.5 $\mu \mathrm{m}$. The photonic crystal defect cavities are formed using electron-beam lithography followed by a series of anistropic dry-etching steps, and a wet chemical etch to undercut the waveguide [4]. An array of defect cavities was fabricated with varying lattice spacings and hole sizes in order to cover a wide range of wavelengths of the defect mode resonance. A scanning electron microsocope image of the laser array is shown in Fig. 2. The defect cavities consist of eight periods of the photonic crystal surrounding a single removed hole in the center. The patterned membrane diameter varied with the lattice spacing but was on average $8 \mu \mathrm{m}$, and the devices were spaced by $10 \mu \mathrm{m}$. The defect cavity lattice spacing was varied from $564 \mathrm{~nm}$ down to $470 \mathrm{~nm}$, and for each different lattice spacing a series of devices with $r / a$ ratios between 0.30 and 0.40 were fabricated.

The sample was mounted on an $X-Y-Z$ stage and the defect cavities were optically pumped from above at an angle normal to the sample surface. An 830-nm semiconductor laser diode was used as the pump source in this experiment, and a $100 \times$ objective lens was used to focus the pump beam to a $4-\mu \mathrm{m}$ spot as well as to collect the photoluminescence (PL). We first measured the PL from a typical defect cavity subthreshold (Fig. 3). The PL is strongly frustrated by the photonic crystal except for two resonance peaks. The narrow peak at $1580 \mathrm{~nm}$ corresponds to emission into a weakly localized shallow "acceptor" (SA) mode with frequency that lies just above the lower-lying band edge ("valence" band edge) of the photonic crystal bandgap. The shorter wavelength peak near $1425 \mathrm{~nm}$ corresponds to emission from a strongly localized pair of degenerate deep "donor" (DD) modes with frequency near midgap of the photonic bandgap.

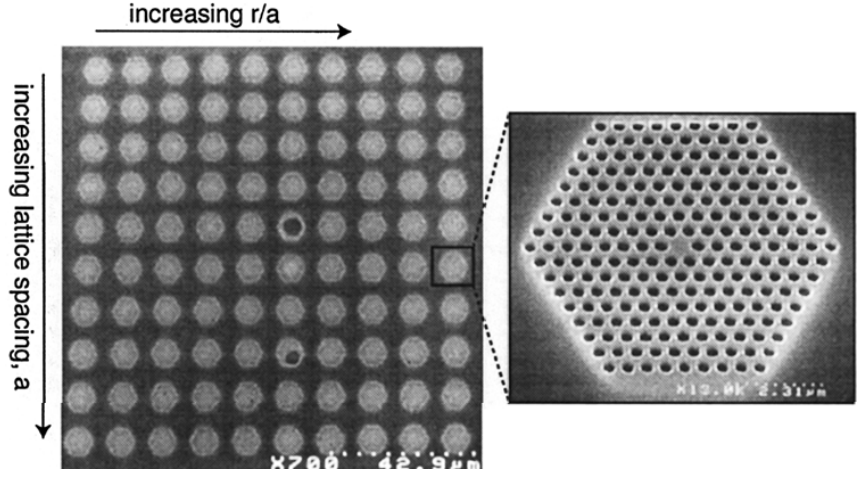

Fig. 2. SEM micrograph of the photonic crystal laser array. The lattice spacing was varied with row number and the hole radius was varied with column number. An expanded view of an individual defect cavity is also shown on the right.

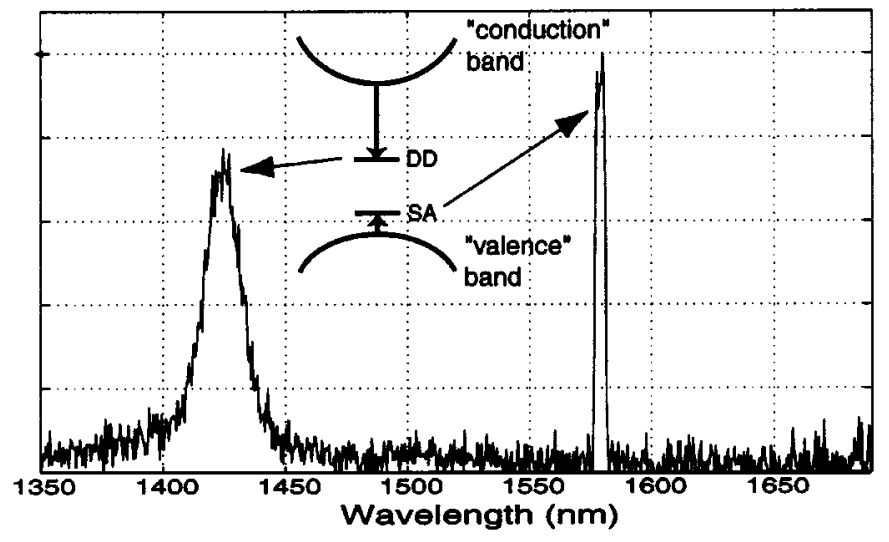

Fig. 3. Near-threshold photoluminescence from a typical defect cavity. The two resonance peaks correspond to the DD and SA cavity modes.

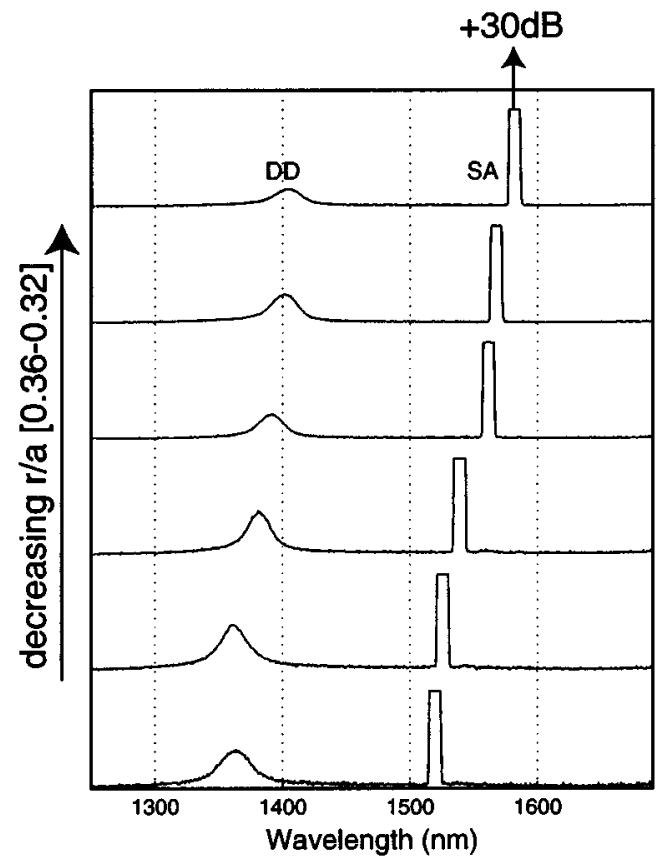

Fig. 4. Tuning of the laser wavelength versus $r / a$. The lattice spacing in this case is fixed at $490 \mathrm{~nm}$, while the radius of the air holes is tuned from $165 \mathrm{~nm}$ to $150 \mathrm{~nm}(r / a=0.38-0.32)$.

The photonic crystal cavity thus acts as a strong mode filter selecting out only two high- $Q$ cavity modes in this case. Details of 


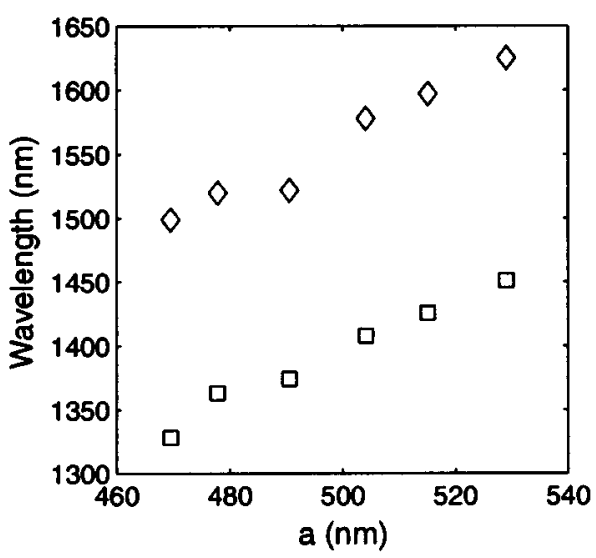

(a)

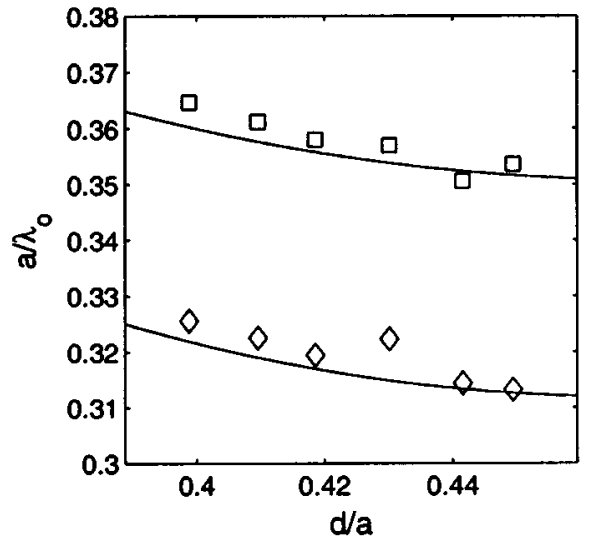

(b)

Fig. 5. Plot of the SA laser peak $(\diamond)$ and the DD peak $(\square)$ for different photonic crystal lattice spacings. (a) Wavelength is plotted versus lattice spacing. (b) Normalized frequency is plotted versus the normalized slab thickness. The solid lines in (b) correspond to FDTD simulations of the DD and SA cavity mode tuning.

the different photonic crystal cavity modes and their properties can be found elsewhere [6].

Lasing was single mode for each cavity tested and only occured off of the longer wavelength SA mode resonance peak at room temperature. This is due to the weak localization and extended nature of the SA mode which results in less vertical diffraction loss and a theoretical $Q$ which is five times that of the strongly localized DD modes. The lasing threshold pump powers were in the $1.5-3 \mathrm{~mW}$ range (external peak pump power), and typical peak fiber coupled output power from the defect laser cavities was about $0.5 \mu \mathrm{W}$. Heating in the undercut membrane limited lasing action to pump pulses shorter than 30 ns (duty cycle $<1 \%$ ) at room temperature.

A plot of the spectra of a series of defect lasers with lattice spacing fixed at $490 \mathrm{~nm}$ and varying hole radius is shown in Fig. 4. The longer wavelength laser line is roughly $30 \mathrm{~dB}$ above the shorter wavelength DD resonance peak. The lasing wave- length tunes between 1520 and $1580 \mathrm{~nm}$ with an interwavelength spacing of $10 \mathrm{~nm}$ as the $r / a$ ratio is varied from 0.38 to 0.32 . This corresponds to a change in the diameter of the air holes of $5 \mathrm{~nm}$ from device to device, which is close to the limit of our lithography system. The DD resonance peak is also seen to tune with $r / a$ as expected.

A series of measurements were also performed on defect cavities with $r / a$ fixed at 0.38 and varying lattice spacings. The defect lasing peak was successfully tuned from 1500 to 1625 $\mathrm{nm}$ with a wavelength spacing of roughly $30 \mathrm{~nm}$. The devices with lattice spacings above $530 \mathrm{~nm}$ did not lase due to the fact that the SA mode is tuned out of the gain bandwidth of the active material. A plot of the wavelength of the SA mode laser peak and DD mode peak versus lattice spacing along with a plot of the corresponding normalized frequency $a / \lambda_{0}$ versus normalized slab thickness $d / a$ is shown in Fig. 5. The normalized frequency slightly decreases with increasing $d / a$ as predicted by (3). Finite-difference time-domain (FDTD) simulations [5] were also performed to model the tuning of the cavity modes, and are shown as solid curves in Fig. 5(b).

\section{CONCLUSION}

We have demonstrated a high-density, multiwavelenth, 2-D laser array at $1.55 \mu \mathrm{m}$ based upon local defect cavities embedded in a 2-D photonic crystal host. A 125-nm laser wavelength tuning range was obtainable through lithographic control of the lattice parameters of the 2-D photonic crystal. A wavelength spacing of $10 \mathrm{~nm}$ between devices was achieved, limited by the tolerances in our etching process. FDTD simulations of the laser cavities is also used to accurately model the tuning of the laser mode.

\section{REFERENCES}

[1] E. Yablonovitch, "Inhibited spontaneous emission in solid-state physics and electronics," Phys. Rev. Lett., vol. 58, no. 20, p. 2059, 1987.

[2] H. Kosaka, T. Kawashima, A. Tomita, M. Notomi, T. Tamamura, T. Sato, and S. Kawakami, "Superprism phenomena in photonic crystals," Phys. Rev. B, vol. 58, no. 16, p. $10096,1998$.

[3] S.-Y. Lin, E. Chow, V. Hietala, P. R. Villeneuve, and J. D. Joannopoulos, "Experimental demonstration of guiding and bending of electromagnetic waves in a photonic crystal," Science, vol. 282, p. 274, 1998.

[4] O. Painter, R. K. Lee, A. Yariv, A. Scherer, J. D. O’Brien, P. D. Dapkus, and I. Kim, "Two-dimensional photonic bandgap defect mode laser," Science, vol. 284, p. 1819, 1999.

[5] O. Painter, J. Vučković, and A. Scherer, "Defect modes of a two-dimensional photonic crystal in an optically thin dielectric slab," J. Opt. Soc. Amer. B, vol. 16, no. 2, p. 275, 1999.

[6] O. Painter, "Donor and acceptor modes of a single defect photonic bandgap laser," in preparation.

[7] J. D. Joannopoulos, R. D. Meade, and J. N. Winn, Photonic Crystals. Princeton, NJ: Princeton Univ. Press, 1995.

[8] I. Kim, D. G. Chang, and P. D. Dapkus, "Growth of $\mathrm{InGaA}_{5} \mathrm{P}$ in a stagnation flow vertical reactor using TBA and TBP," J. Cryst. Growth, vol. 119, no. $1-4$, p. 138 , Dec. 1988. 